\title{
Harmonic oscillations of neutral particles in the $\gamma$ metric
}

\author{
Bobir Toshmatov, 1,2, 用 Daniele Malafarina, ${ }^{1,7}$ and Naresh Dadhich ${ }^{3}$, f \\ ${ }^{1}$ Department of Physics, Nazarbayev University, 53 Kabanbay Batyr, 010000 Astana, Kazakhstan \\ ${ }^{2}$ Ulugh Beg Astronomical Institute, Astronomicheskaya 33, Tashkent 100052, Uzbekistan \\ ${ }^{3}$ Inter-University Centre for Astronomy and Astrophysics, Post Bag 4, Ganeshkhind, Pune 411 007, India
}

\begin{abstract}
We consider a well-known static, axially symmetric, vacuum solution of Einstein equations belonging to Weyl's class and determine the fundamental frequencies of small harmonic oscillations of test particles around stable circular orbits in the equatorial plane. We discuss the radial profiles of frequencies of the radial, latitudinal (vertical), and azimuthal (Keplerian) harmonic oscillations relative to the comoving and distant observers and compare with the corresponding ones in the Schwarzschild and Kerr geometries. We show that there exist latitudinal and radial frequencies of harmonic oscillations of particles moving along the circular orbits for which it is impossible to determine whether the central gravitating object is described by the slowly rotating Kerr solution or by a slightly deformed static space-time.
\end{abstract}

\section{INTRODUCTION}

The Zipoy-Vorhees space-time, also known as $\gamma$ metric is an asymptotically flat vacuum solution of Einstein's equations which belongs to the Weyl class of static, axially symmetric space-times [1, 2]. The $\gamma$ metric is completely characterized by two parameters, namely $M>0$, which is related to the gravitational mass of the source and the deformation parameter $\gamma>0$. In the case of $\gamma=1$, the Schwarzschild space-time is recovered. On the other hand, the cases of $\gamma>1$ and $\gamma<1$ correspond to oblate and prolate spheroidal sources, respectively, thus, showing that the parameter $\gamma$ can be considered as a deformation parameter and that for $\gamma \neq 1$ the coordinates are not spherical.

From the no-hair theorem it is obvious that for $\gamma \neq 1$ the line element does not describe a black hole. In fact, it can be shown that the surface $r=2 M$ corresponds to a genuine curvature singularity for every value of $\gamma$ different from

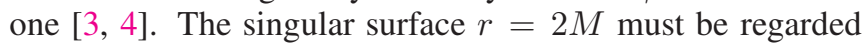
as an infinitely redshifted surface which observationally may present features similar to the Schwarzschild event horizon. However, for certain values of $\gamma$, the spacetime presents some unique features that allow it to be distinguished from the Schwarzschild solution.

For these reasons, the $\gamma$ metric can be considered as a black hole "mimicker" and, being an exact solution of Einstein's equations, constitutes an excellent candidate to study possible astrophysical tests of black hole space-times.

The geometrical properties of the $\gamma$-metric have been studied in [5] 8 while interior solutions have been found in 11]. The motion of test particles and light rays in the $\gamma$ metric has been studied in $15,12,15$.

The frequencies of quasiperiodic oscillations in the Schwarzschild metric were studied for example in [16, 17, while for the Kerr metric they have been studied in various physical scenarios, for example in [18 20]. The application

\footnotetext{
bobir.toshmatov@nu.edu.kt

daniele.malafarina@nu.edu.kz

$\ddagger$ nkd@iucaa.in
}

to astrophysics and the study of astrophysical black holes was discussed in $21-27$.

In the present article we study the frequencies of small harmonic oscillations of test particles about stable circular orbits in the $\gamma$ metric and compare them with the corresponding frequencies in the Schwarzschild and Kerr space-times. We find that differences appear at small radii (either approaching the innermost stable circular orbit (ISCO) or approaching the infinitely redshifted surface) and a combination of measurements for epicyclic frequencies at small radii around compact objects could be used in principle to determine the nature of its geometry.

The paper is organized as follows: In Sec. II we recap the equations describing the motion of test particles in the $\gamma$ metric and derive the values of $\gamma$ that separate different behaviours. Sec. III is devoted to the study of small harmonic oscillations for test particles about the circular geodesics. Finally in Sec. IV the results are summarized and put in the context of possible future astrophysical observations of black holes. Throughout the paper we make use of natural units setting $G=c=1$.

\section{DYNAMICS OF TEST PARTICLES}

In Erez-Rosen coordinates [28] the $\gamma$ metric is represented by the line element

$$
\begin{aligned}
d s^{2}= & -f^{\gamma} d t^{2}+f^{\gamma^{2}-\gamma} g^{1-\gamma^{2}}\left(\frac{d r^{2}}{f}+r^{2} d \theta^{2}\right)+ \\
& +f^{1-\gamma} r^{2} \sin ^{2} \theta d \phi^{2}
\end{aligned}
$$

where

$$
\begin{aligned}
& f(r)=1-\frac{2 M}{r}, \\
& g(r, \theta)=1-\frac{2 M}{r}+\frac{M^{2} \sin ^{2} \theta}{r^{2}} .
\end{aligned}
$$

From the asymptotic expansion of the gravitational potential it is easy to see that the total mass of the source as measured by an observer at infinity is $M_{\text {tot }}=M \gamma$ 㠼. Also from evaluation of the Kretschmann scalar it is possible to see that the surface $r=2 M$ is a true curvature singularity for 
all values of $\gamma \neq 1$ [3]. 1] Therefore the radial coordinate in the $\gamma$ space-time takes values $r \in(2 M, \infty)$. If we understand the singularity as the regime at which the classical description fails, then we can interpret the surface $r=2 \mathrm{M}$ as the boundary of an exotic compact object that is intrinsically quantum-gravitational in nature. Our purpose is to investigate the properties of test particles orbiting around such an exotic compact object and determine whether they can in principle be distinguished from the corresponding cases around a black hole.

\section{A. Equations of motion}

Since the space-time under study does not depend explicitly on time, the Hamiltonian plays the role of the total energy of the system and governs the dynamics of neutral test particle. Such Hamiltonian for test particles in curved space-time can written as

$$
H=\frac{1}{2} g^{\mu \nu} p_{\mu} p_{\nu}+\frac{1}{2} m^{2},
$$

where $p^{\mu}$ is four-momentum that is defined as $p^{\mu}=m u^{\mu}$ with $m$ and $u^{\mu}$ being mass and four-velocity of the test particle, respectively. From Noether's theorem, as the system does not depend explicitly on the coordinates $t$ and $\phi$, we know that the associated conjugate momenta, namely the energy $E$ and angular momentum $L$ are conserved and

$$
p_{t}=g_{t t} \frac{d t}{d \tau}=-E, \quad p_{\phi}=g_{\phi \phi} \frac{d \phi}{d \tau}=L .
$$

From Eq. (4) one can find the $t$ and $\phi$ components of the fourvelocity of the test particle as

$$
\frac{d t}{d \tau}=-g^{t t} E, \quad \frac{d \phi}{d \tau}=g^{\phi \phi} L
$$

and thus we obtain

$$
H=\frac{1}{2} g^{r r} p_{r}^{2}+\frac{1}{2} g^{\theta \theta} p_{\theta}^{2}+H_{t \phi},
$$

with

$$
H_{t \phi}(r, \theta)=\frac{1}{2}\left(g^{t t} E^{2}+g^{\phi \phi} L^{2}+m^{2}\right) .
$$

The normalization condition $u_{\mu} u^{\mu}=-1$ leads to $H=0$. Then, from Eq. (6) one finds that

$$
g_{r r}\left(\frac{d r}{d \tau}\right)^{2}+g_{\theta \theta}\left(\frac{d \theta}{d \tau}\right)^{2}=-\frac{2 H_{t \phi}(r, \theta)}{m^{2}} .
$$

If one considers a particle moving on a plane with $\theta_{0}=$ const., as is the case for particles in accretion disks that are

\footnotetext{
${ }^{1}$ However it is also an infinite red-shift surface which means any signal emanating from it would be infinitely red-shifted. It would therefore be rather innocous for an external observer.
}

confined near the equatorial plane $\theta=\pi / 2$, then the equation of motion (8) takes the form

$$
g_{r r}\left(\frac{d r}{d \tau}\right)^{2}=R(r) \equiv-\frac{2 H_{t \phi}\left(r, \theta_{0}\right)}{m^{2}},
$$

where radial function $R(r)$ can be written as

$$
R(r)=\left(1-\frac{2 M}{r}\right)^{-\gamma}\left(E^{2}-V_{e f f}(r)\right),
$$

with

$$
V_{e f f}(r)=\frac{L^{2}}{r^{2}}\left(1-\frac{2 M}{r}\right)^{2 \gamma-1}+\left(1-\frac{2 M}{r}\right)^{\gamma}
$$

If, on the other hand, the particle is moving along a circular orbit on $r=r_{0}$ with $\theta \neq$ const., then the equation of motion (8) takes the form

$$
g_{\theta \theta}\left(\frac{d \theta}{d \tau}\right)^{2}=\Theta(\theta) \equiv-\frac{2 H_{t \phi}\left(r_{0}, \theta\right)}{m^{2}},
$$

By combining the two conditions above, for a particle on circular orbit $r=r_{0}$ in the plane $\theta=\theta_{0}$, we obtain

$$
R\left(r_{0}\right)=0,\left.\quad \partial_{r} R(r)\right|_{r_{0}}=0,
$$

(or $V_{\text {eff }}\left(r_{0}\right)=E^{2}$ and $\partial_{r} V_{\text {eff }}\left(r_{0}\right)=0$ ) and

$$
\Theta\left(\theta_{0}\right)=0,\left.\quad \partial_{\theta} \Theta(\theta)\right|_{\theta_{0}}=0,
$$

\section{B. Circular orbits}

In the following we shall focus on the radii of characteristic circular orbits in the equatorial plane of the $\gamma$ space-time, since these are the most relevant orbits for astrophysical purposes, as they describe the motion of particles of gas in accretion disks around compact objects.

By solving Eqs. (13), simultaneously, one finds the specific energy $E=E / m$ and the specific angular momentum $L=$ $L / m$ of test particles moving along circular orbits as

$$
\begin{aligned}
& E=-\frac{g_{t t}}{\sqrt{-\left(g_{t t}+g_{\phi \phi} \Omega^{2}\right)}}, \\
& L=\frac{g_{\phi \phi} \Omega}{\sqrt{-\left(g_{t t}+g_{\phi \phi} \Omega^{2}\right)}},
\end{aligned}
$$

Here $\Omega=d \phi / d t$ is the angular velocity of the test particle as measured by distant observers and it is given by

$$
\Omega= \pm \sqrt{-\frac{g_{t t, r}}{g_{\phi \phi, r}}} .
$$

Thus, we rewrite expressions $15,(16)$ and $(17)$ in terms of the $\gamma$-metric as

$$
\begin{aligned}
& E=\left(1-\frac{2 M}{r}\right)^{\gamma / 2} \sqrt{\frac{r-\gamma M-M}{r-2 \gamma M-M}}, \\
& L= \pm r\left(1-\frac{2 M}{r}\right)^{(1-\gamma) / 2} \sqrt{\frac{M \gamma}{r-M-2 M \gamma}}, \\
& \Omega= \pm\left(1-\frac{2 M}{r}\right)^{\gamma-1 / 2} \frac{1}{r} \sqrt{\frac{M \gamma}{r-M-M \gamma}} .
\end{aligned}
$$


From the above expressions one can see that the regions of the $\gamma$ space-time where test particles can have circular orbits are given by:

$$
\begin{aligned}
& \text { (i) } r>M(2 \gamma+1) \text { if } \gamma \geq \frac{1}{2}, \\
& \text { (ii) } r>2 M \text { if } 0<\gamma<\frac{1}{2} .
\end{aligned}
$$

Moreover, from the fact that at the light ring (photon's capture orbits) the specific energy (18) diverges because photon's mass is zero and the relevant parameter is the ratio, $L / E$, one can easily find the location of photon capture orbit as

$$
r_{p s}=M(2 \gamma+1), \quad \text { with } \quad \gamma \geq \frac{1}{2},
$$

while for $\gamma<1 / 2$ no photon capture orbit is present. By comparing (21) with (22) one can say that innermost positions of circular orbits of test particles are limited by the circular geodesics of massless particles. That is, photon circular orbit defines the existence threshold, $r>r_{p s}$, for timelike circular orbits. Further note that $r_{p s}$ is always preceded by unstable circular orbits.

Circular orbits with $r>r_{p s}$ are therefore unstable and slight departures from circularity leads to unbound motion. Unbound orbits are separated from the bound orbits by a critical geodesic called the marginally bound circular orbit $r_{m b}$ that is found from the zero binding energy $E_{\text {bind }} \equiv$ $E(\infty)-E\left(r_{m b}\right)=0$ [29, 30]. Since the $\gamma$ space-time is asymptotically flat, $E(\infty)=1$ therefore, $E\left(r_{m b}\right)=1$ gives

$$
r_{m b}-M-2 M \gamma-\left(r_{m b}-M-M \gamma\right)\left(1-\frac{2 M}{r_{m b}}\right)^{\gamma}=0 \text {, }
$$

A particle with energy $E>1$ that slightly departs from circular orbit has unbound motion. This means that the circular orbit is unstable in such a way that with an infinitesimal small outward perturbation, the particle will escape to infinity on an asymptotically hyperbolic trajectory. On the other hand, for $E<1$, perturbing a particle on an unstable circular orbit would lead to bound motion. In the case of $\gamma=1$, by solving Eq. (23), one obtains the marginally bound circular orbit in the Schwarzschild space-time, $r_{m b}=4 M$.

Now we find the marginally stable circular orbits, also called innermost stable circular orbits (ISCO). All the stable circular orbits of test particles (with radius $r_{s t}$ ) satisfy the condition $\left(V_{e f f}^{\prime \prime}\left(r_{s t}\right) \geq 0\right.$ ) or

$$
\begin{aligned}
& \left.R^{\prime \prime}(r)\right|_{r_{s t}}= \\
& \frac{2 M \gamma\left[r_{s t}^{2}-2 M(1+3 \gamma) r_{s t}+2 M^{2}(1+2 \gamma)(1+\gamma)\right]}{r_{s t}^{2}\left(r_{s t}-2 M\right)\left(M+2 M \gamma-r_{s t}\right)} \leq 0,
\end{aligned}
$$

with the equality holding for the smallest allowed value for stable circular orbits, namely the ISCO. By solving the equation $R^{\prime \prime}(r)=0$ (or $V^{\prime \prime}(r)=0$ ), one finds two roots for the ISCO given by

$$
r_{i s c o, \pm}=M\left(1+3 \gamma \pm \sqrt{5 \gamma^{2}-1}\right) .
$$

Once again, for $\gamma=1$ we retrieve $r_{i s c o,+}=6 M$ which is the value of the ISCO for the Schwarzschild geometry. Here it is easy to notice that for $\gamma=1 / \sqrt{5}$, the two values of the ISCO coincide. Moreover, for $\gamma \geq 1 / \sqrt{5}$ the value of $r_{i s c o,+}$ is always greater than the bounds imposed by conditions (21) and therefore there is always at least one marginally stable circular orbit. However, $r_{i s c o,-}$ does not satisfy the bounds imposed by conditions (21) for values $\gamma \in(1 / 2, \infty)$. Although it is natural to expect that stable circular orbits will be allowed at great distances and cease to exist at a certain distance from the source, the above discussion shows that in the range $\gamma \in[1 / \sqrt{5}, 1 / 2]$ there exist a second range of stable circular orbits closer to center (see Fig. 1). We can understand better the reason for this behaviour by analyzing the effective potential in eq. (11) term by term. It is easy to see that at small radii the term proportional to $L^{2} / r^{2}$ will dominate over the other terms. Then, as $\gamma<1 / 2$ the term $(1-2 M / r)^{2 \gamma-1}$ will go at the denominator causing the change in the behaviour of the effective potential. This new region where stable circular orbits are allowed extends from $r=2 M$ until a finite distance, determined by the second root of the ISCO equation. However, for $\gamma=1 / \sqrt{5}$ the two values of the ISCO radii coincide and for $\gamma<1 / \sqrt{5}$ stable circular orbits are allowed at any distance from the source, similarly to the Newtonian case.

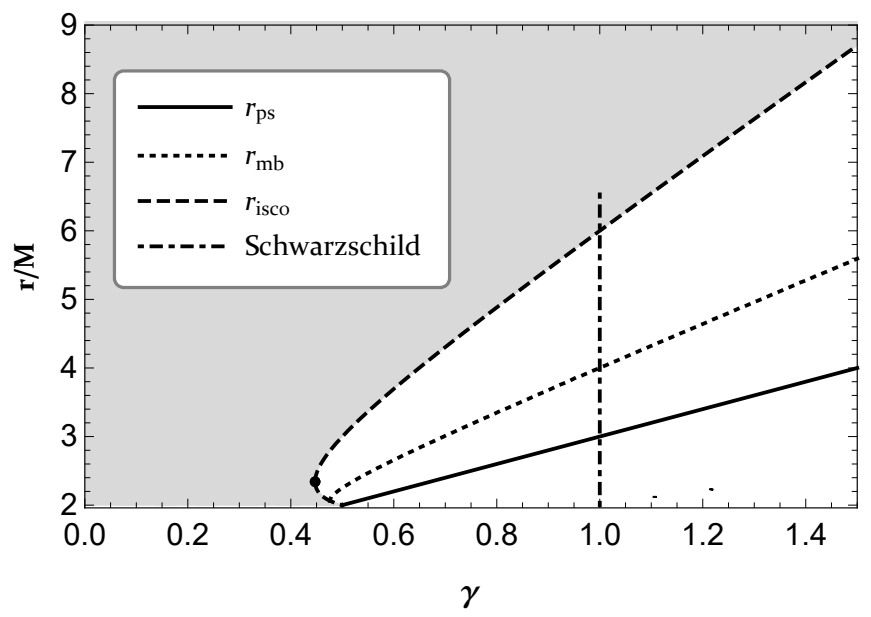

FIG. 1. Dependence of radii of characteristic circular orbits: light ring $\left(r_{p s}\right.$-black, solid), marginally bound orbit $\left(r_{m b}\right.$-black, dotted), and ISCO ( $r_{\text {isco }}$-black, dashed) in the $\gamma$ metric as functions of $\gamma$. Here the vertical dot-dashed line corresponds to the values for the Schwarzschild space-time with $\gamma=1$. The curve for $r_{i s c o,-}$ for $\gamma>1 / 2$ is not shown since marginally stable circular orbits are not allowed in this case. The gray region corresponds to the range of values of $r$ and $\gamma$ where stable circular orbits are allowed.

In order to extract more information on the region of the stable circular orbits we can plot the effective potential $V_{\text {eff }}(r)$ in eq. 11) for different values of $\gamma \in(0,1 / 2)$ (see Fig. 2 for the case of $\gamma \in(1 / \sqrt{5}, 1 / 2))$. Depending on the value of $\gamma$ the effective potential $V_{\text {eff }}$ can have up to two minima and one maximum. From the condition (24) one can easily realize that maxima (minima) of the radial function (effective potential) correspond to stable circular orbits. Then, the outer edge 

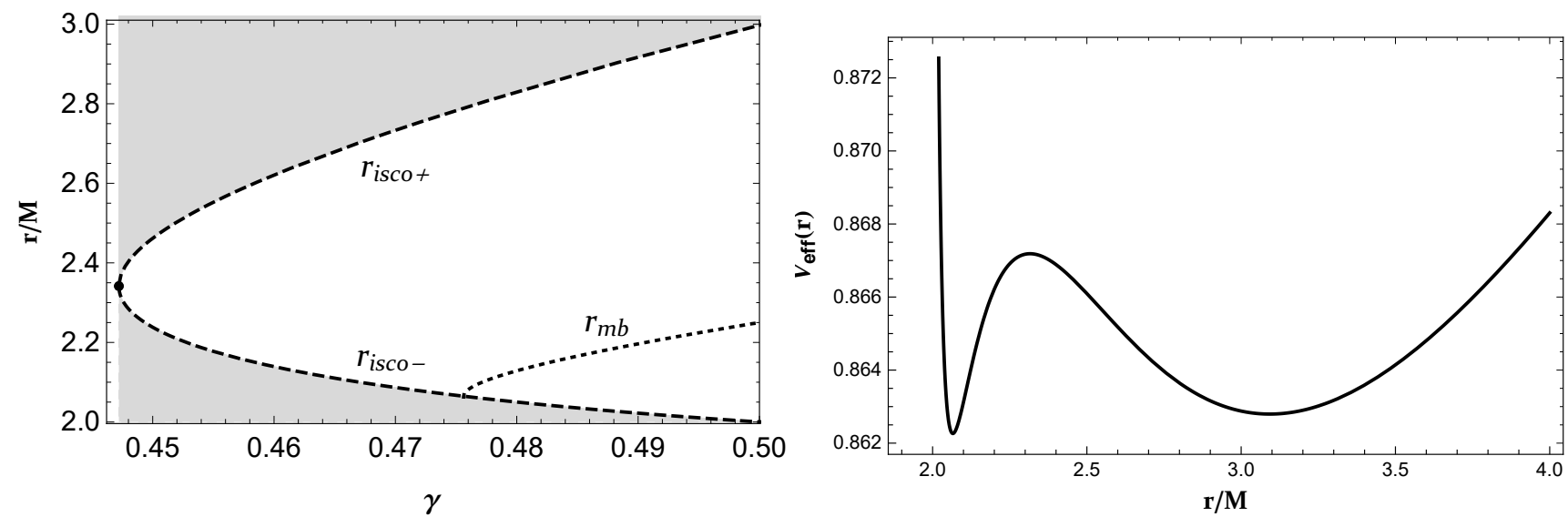

FIG. 2. Left panel: Boundary of the ISCO radii for $\gamma \in[1 / \sqrt{5}, 1 / 2]$ from Fig. 1. Here the gray shaded region represents the range of radii for which stable circular orbits can exist as function of $\gamma$, while the white region corresponds to the range for which no stable circular orbits exist. The value of $r_{m b}$ separates the regime where unstable orbits are bound from that were they are unbound. Right panel: Radial profile of the effective potential $V_{\text {eff }}$ in eq. 11) for $\gamma \in(1 / \sqrt{5}, 1 / 2)$. There are two minima of $V_{\text {eff }}$ corresponding to the stable circular orbits (where $V_{e f f}^{\prime \prime}\left(r_{s t}\right) \geq 0$ ), and one maximum corresponding to the unstable circular orbit (where $V_{\text {eff }}^{\prime \prime}\left(r_{u n s t}\right)<0$ ).

of the inner stable circular orbits corresponds to $r_{i s c o-}$, while the inner edge of the outer stable circular orbits corresponds to $r_{i s c o+}$, i.e. the two solutions of the ISCO equation, respectively. The maximum of the effective potential corresponds to the unstable circular orbits. The effective potential exhibits this behaviour with two minima and and one maximum precisely for $1 / \sqrt{5}<\gamma<1 / 2-$ see right panel of Fig. 2. This shows that in two disjoint regions $r_{s t} \in\left(2 M, r_{i s c o-}\right]$ and $r_{s t} \in\left[r_{i s c o+}, \infty\right)$ the circular orbits are stable. However, in the region between these, i.e. $r_{\text {unst }} \in\left(r_{i s c o-}, r_{i s c o+}\right)$, no stable circular orbits can exist. For $\gamma>1 / 2$, the behaviour of the radial function (10) becomes similar to the Schwarzschild case, where stable circular orbits are possible only for $r_{s t} \in\left[r_{i s c o+}, \infty\right)$. At $\gamma=\sqrt{5}$ the outer and inner minima merge and for $\gamma \leq 1 / \sqrt{5}$ the behaviour of the radial function resembles the Newtonian case and stable circular orbits are allowed everywhere. This suggests that the term responsible for the angular momentum interaction with the mass (the term $L^{2} M / r^{3}$ in the Schwarzschild case) behaves qualitatively similar to the Schwarzschild case for $\gamma>1 / 2$, namely it is attractive causing the stable circular orbits to cease to exist at a certain radius, while it behaves in the opposite way for $\gamma \leq 1 / 2$. The interaction between angular momentum and mass turns from attractive to repulsive as $\gamma$ goes from $\gamma>1 / 2$ to $\gamma<1 / 2$. This can be clearly seen by studying the term

$$
W(r)=\frac{L^{2}}{r^{2}}\left(1-\frac{2 M}{r}\right)^{2 \gamma-1},
$$

in the effective potential (11). It is immediately seen for $\gamma=1 / 2$ we have $W=L^{2} / r^{2}$ and therefore the relativistic correction to the Newtonian behaviour vanishes. Then from the fact that $W^{\prime}(r)=d W / d r$ near $r=2 M$ changes sign at $\gamma=1 / 2$ we see that $W$ changes from increasing (attractive) for $\gamma>1 / 2$ to decreasing (repulsive) for $\gamma<1 / 2$ in the vicinity of the singularity.

Thus, taking into account the conditions (21), we can conclude that the stable circular orbits can exist in different re- gions as follows:

$$
\begin{aligned}
& r \in\left[r_{i s c o+},+\infty\right) \quad \text { for } \quad \gamma \in[1 / 2,+\infty), \\
& r \in\left(2 M, r_{i s c o-}\right] \cup\left[r_{i s c o+},+\infty\right) \text { for } \gamma \in[1 / \sqrt{5}, 1 / 2), \\
& r \in(2 M,+\infty) \text { for } \gamma \in(0,1 / \sqrt{5}) .
\end{aligned}
$$

It is interesting to notice that through the behaviour of the motion of test particles we can gain some insight on the nature of the curvature singularity in the space-time. In the case of Schwarzschild, the singularity located at $r=0$ is attractive and particles are crushed in it by diverging tidal forces. Similarly, in the case of the $\gamma$ metric we can see that the same behaviour occurs at $r=2 M$ only for large values of $\gamma$. This can be seen for example from the Kretschmann scalar $K$, which behaves like $1 /(r-2 M)^{6}$ for $\gamma=2$. Therefore if we characterize the strength of the singularity by the exponent $s$ for which $K \simeq 1 /(r-2 M)^{s}$, we see that $s>6$ for $\gamma>2$, $s>4$ for $\gamma>1+\sqrt{5}$, and $s<2$ for $\gamma<1$, suggesting that for prolate sources the singularity is weaker than in the Schwarzschild case. For $\gamma>1 / 2$ the singularity is 'attractive' as can be seen from the fact that the interaction between mass and angular momentum vanishes at $r=2 M$. On the other hand, when $\gamma<1 / 2$, the interaction term between the mass and the angular momentum of the test particle is repulsive and blows up at $r=2 M$, as in the case of the monopolequadrupole solution of the Weyl class, which also describes small deviations from spherical symmetry [31]. Therefore, in this case, the singularity is "repulsive" and test particles will be ejected rather than crushed.

\section{HARMONIC OSCILLATIONS OF NEUTRAL TEST PARTICLE}

In this section we will study the motion of test particles that slightly depart from circular orbit. As we mentioned in the previous section, stable circular orbits for test particles in the 
$\gamma$ space-time are located at a radius $r_{0}$ and latitudinal angle $\theta_{0}=\pi / 2$ corresponding to the minimum of the function $H_{t \phi}$ (or $R(r)$ and $\Theta(\theta)$, respectively) given in Eq. (7). If a test particle deviates slightly from the stable circular orbit it will start to oscillate around its equilibrium value performing an epicyclic motion. This oscillating motion is governed by the epicyclic frequencies.

\section{A. Epicyclic frequencies}

For the sake of clarity we shall restrict the analysis to the linear regime, and consider separately the case of purely radial, i.e. $r=r_{0}+\delta r, \delta \theta=0$, and purely vertical,i.e. $\theta=\theta_{0}+\delta \theta, \delta r=0$, epicyclic oscillations about circular orbits in the equatorial plane $\theta_{0}=\pi / 2$. If we write the Taylor expansion of the functions $R(r)$ and $\Theta(\theta)$ in powers of $\delta r$ and $\delta \theta$, respectively as

$$
\begin{aligned}
& R(r)=R\left(r_{0}\right)+\left.\partial_{r} R(r)\right|_{r_{0}} \delta r+\left.\frac{1}{2} \partial_{r}^{2} R(r)\right|_{r_{0}} \delta r^{2}+\ldots \\
& \Theta(\theta)=\Theta\left(\theta_{0}\right)+\left.\partial_{\theta} \Theta(\theta)\right|_{\theta_{0}} \delta \theta+\left.\frac{1}{2} \partial_{\theta}^{2} \Theta(\theta)\right|_{\theta_{0}} \delta \theta^{2}+\ldots
\end{aligned}
$$

and apply the circularity conditions (13) and (14), we arrive at

$$
\begin{aligned}
& g_{r r} \delta \dot{r}^{2}=\left.\frac{1}{2} \partial_{r}^{2} R(r)\right|_{r_{0}} \delta r^{2}, \\
& g_{\theta \theta} \delta \dot{\theta}^{2}=\left.\frac{1}{2} \partial_{\theta}^{2} \Theta(\theta)\right|_{\theta_{0}} \delta \theta^{2} .
\end{aligned}
$$

Considering that the total energy of the orbit is conserved, one finds that

$$
\begin{aligned}
& \delta \dot{r}\left[g_{r r} \delta \ddot{r}-\left.\frac{1}{2} \partial_{r}^{2} R(r)\right|_{r_{0}} \delta r\right]=0, \\
& \delta \dot{\theta}\left[g_{\theta \theta} \delta \ddot{\theta}-\left.\frac{1}{2} \partial_{\theta}^{2} \Theta(\theta)\right|_{\theta_{0}} \delta \theta\right]=0,
\end{aligned}
$$

Where $\delta \dot{r}=0$ and $\delta \dot{\theta}=0$ are the trivial solutions corresponding to no oscillations. It is immediately apparent that the parts within the square brackets of the above equations are in the form of harmonic oscillators as 16,32

$$
\begin{aligned}
& \delta \ddot{r}+\omega_{r}^{2} \delta r=0, \\
& \delta \ddot{\theta}+\omega_{\theta}^{2} \delta \theta=0,
\end{aligned}
$$

with

$$
\begin{aligned}
& \omega_{r}^{2}=-\frac{\left.\partial_{r}^{2} R(r)\right|_{r_{0}}}{2 g_{r r}}=\frac{\left.\partial_{r}^{2} H_{t \phi}\right|_{r_{0}}}{g_{r r}}, \\
& \omega_{\theta}^{2}=-\frac{\left.\partial_{\theta}^{2} \Theta(\theta)\right|_{\theta_{0}}}{2 g_{\theta \theta}}=\frac{\left.\partial_{\theta}^{2} H_{t \phi}\right|_{\theta_{0}}}{g_{\theta \theta}},
\end{aligned}
$$

where the specific energy and angular momentum of the test particles on circular orbits are given by Eq. (18) and Eq. (19), respectively. Therefore we can obtain the explicit forms of the frequencies as

$$
\begin{aligned}
& \omega_{r}^{2}=\left(1-\frac{M}{r}\right)^{2 \gamma^{2}-2}\left(1-\frac{2 M}{r}\right)^{-\gamma^{2}+\gamma-1} \frac{M \gamma\left[r^{2}-2(3 \gamma+1) M r+2(\gamma+1)(2 \gamma+1) M^{2}\right]}{r^{4}(r-M-2 M \gamma)}, \\
& \omega_{\theta}^{2}=\left(1-\frac{M}{r}\right)^{2 \gamma^{2}-2}\left(1-\frac{2 M}{r}\right)^{-\gamma^{2}+\gamma} \frac{M \gamma}{r^{2}(r-M-2 M \gamma)},
\end{aligned}
$$

By inspecting expression (37) one can notice that zeros of expression in the square bracket correspond to the radii of the ISCO as given in Eq. (25). Thus, we see that radial epicyclic frequency vanishes at the ISCO, $\omega_{r}\left(r_{i s c o}\right)=0$. This is reasonable, considering the fact that the ISCO is a marginally stable orbit and that below ISCO, at least for values of $\gamma>1 / 2$, the particles start to fall towards the central object, and no radial oscillations occur.

Moreover, there is another very important angular frequency of circular epicyclic motion of the particle, namely the azimuthal (or Keplerian) frequency of the circular motion in the equatorial plane, defined by the relation

$$
\omega_{\phi}^{2}=\dot{\phi}^{2}=\left(1-\frac{2 M}{r}\right)^{\gamma-1} \frac{M \gamma}{r(r-M-2 M \gamma)} .
$$

Note that the frequencies $\omega_{r}, \omega_{\theta}$ and $\omega_{\phi}$ are measured with respect to the proper time of a comoving observer. To get the observed frequencies by a observer at infinity, one needs to divide them by the square of the redshift factor $u^{t}$ as

$$
\Omega_{r(\theta, \phi)}^{2}=\frac{\omega_{r(\theta, \phi)}^{2}}{\left(u^{t}\right)^{2}},
$$

where squared redshift factor for the $\gamma$ metric is given by

$$
\left(u^{t}\right)^{2}=\left(1-\frac{2 M}{r}\right)^{-\gamma} \frac{r-M-M \gamma}{r-M-2 M \gamma} .
$$

Thus, frequencies (37), (38) and (39) take the following form when they are measured by observers at infinity: 


$$
\begin{aligned}
& \Omega_{r}^{2}=\left(1-\frac{M}{r}\right)^{2 \gamma^{2}-2}\left(1-\frac{2 M}{r}\right)^{-(\gamma-1)^{2}} \frac{M \gamma\left[r^{2}-2(3 \gamma+1) M r+2(\gamma+1)(2 \gamma+1) M^{2}\right]}{r^{4}(r-M-M \gamma)}, \\
& \Omega_{\theta}^{2}=\left(1-\frac{M}{r}\right)^{2 \gamma^{2}-2}\left(1-\frac{2 M}{r}\right)^{-\gamma^{2}+2 \gamma} \frac{M \gamma}{r^{2}(r-M-M \gamma)}, \\
& \Omega_{\phi}^{2}=\left(1-\frac{2 M}{r}\right)^{2 \gamma-1} \frac{M \gamma}{r^{2}(r-M-M \gamma)} .
\end{aligned}
$$

In Fig. 3 we show the dependence of the epicyclic frequencies for neutral test particles about the radii of stable circular orbits for different values of $\gamma$. One can see that the azimuthal frequencies are monotonically decreasing functions of the radius. More in detail, depending on the value of the parameter $\gamma$ the epicyclic frequencies as measured by observers at spatial infinity behave as follows:

(i) If $\gamma>1$, the latitudinal and azimuthal epicyclic frequencies diverge, $\Omega_{\theta}, \Omega_{\phi} \rightarrow \infty$, at $r=M(1+\gamma)$; the radial epicyclic frequency vanishes at the radius of the outer ISCO, namely $\Omega_{r}=0$ at $r=r_{i s c o,+}$ (see first panel of Fig. 3).

(ii) If $\gamma=1$, the latitudinal and azimuthal epicyclic frequencies diverge, $\Omega_{\theta}, \Omega_{\phi} \rightarrow \infty$, at $r=0$; the radial epicyclic frequency vanishes at the radius of ISCO, namely $\Omega_{r}=0$ at $r=r_{i s c o,+} \equiv 6 M$. These are the known results for the Schwarzschild space-time.

(iii) If $1 / 2<\gamma<1$, the latitudinal and azimuthal epicyclic frequencies vanish, $\Omega_{\theta}=\Omega_{\phi}=0$, at $r=2 M$; the radial epicyclic frequency vanishes at the radius of ISCO, namely $\Omega_{r}=0$, at $r=r_{i s c o,+}$ (see second panel of Fig. (3).

(iv) If $1 / \sqrt{5} \leq \gamma \leq 1 / 2$, the latitudinal frequency vanishes, $\Omega_{\theta}=0$ at $r=2 M$, while the azimuthal frequency diverges, $\Omega_{\phi} \rightarrow \infty$ at $r=2 M$; the radial frequency vanishes at the extremal ISCO, namely $\Omega_{r}=0$ at $r=$ $(1+3 / \sqrt{5}) M$, and then monotonically increases with smaller radii to diverge at the singularity, namely $\Omega_{r} \rightarrow$ $\infty$ at $r=2 M$ (see third panel of Fig. 3).

(v) If $0<\gamma<1 / \sqrt{5}$, the latitudinal epicyclic frequency vanishes, $\Omega_{\theta}=0$, at $r=2 M$, while the radial and azimuthal epicyclic frequencies diverge, $\Omega_{r}, \Omega_{\phi} \rightarrow \infty$, at $r=2 M$. Notice that there is no ISCO in this range of values for $\gamma$ (see fourth panel of Fig. 3).

Small changes in value of the parameter $\gamma$ do not affect much the azimuthal, $\Omega_{\phi}$, and latitudinal, $\Omega_{\theta}$, frequencies, how- ever, the effects on the radial frequency, $\Omega_{r}$, are more significant. However, a striking difference appears in the longitudinal and azimuthal frequencies, when we compare with the Schwarzschild case. In fact, for $\gamma=1$ both $\Omega_{\theta}$ and $\Omega_{\phi}$ have finite, non vanishing value at $r=2 M$, while for $\gamma<1$ they either diverge or vanish.

The local extrema of the epicyclic frequencies are given by the condition

$$
\frac{\partial \Omega_{i}}{\partial r}=0
$$

where $i=r, \theta, \phi$. For the values $\gamma \geq 1 / \sqrt{5}$ radial frequency always has local maximum close to the outer ISCO radius, $r_{i s c o,+}$, and this maximum value increases with decreasing value of $\gamma$. Thus, when $\gamma=1 / \sqrt{5}$, the radial epicyclic frequency of the $\gamma$ spacetime reaches the possible highest maximum value, $\Omega_{r}=0.0044 / M$ at $r=(1+3 / \sqrt{5}) M$.

It is well known that at large distances the Schwarzschild space-time has Newtonian behaviour and for Newton all the three components of epicyclic frequencies have the same value given by [33]

$$
\Omega_{r}^{2}=\Omega_{\theta}^{2}=\Omega_{\phi}^{2}=\frac{M}{r^{3}} .
$$

As expected, at large distances the epicyclic frequencies in the $\gamma$ metric exhibit Newtonian behaviour as

$$
\Omega_{r}^{2}=\Omega_{\theta}^{2}=\Omega_{\phi}^{2}=\frac{M \gamma}{r^{3}}+O\left(\frac{1}{r^{4}}\right)
$$

which can be interpreted as a further confirmation that the total mass of the $\gamma$ space-time as measured by distant observers is $M_{\text {tot }}=M \gamma$.

In order to estimate the effects due to the departure from spherical symmetry of the space-time, we can study how small deviations from the Schwarzschild metric affect the epicyclic frequencies. By considering $\gamma=1+\epsilon$ with $|\epsilon| \ll 1$, the epicyclic frequencies take the form 

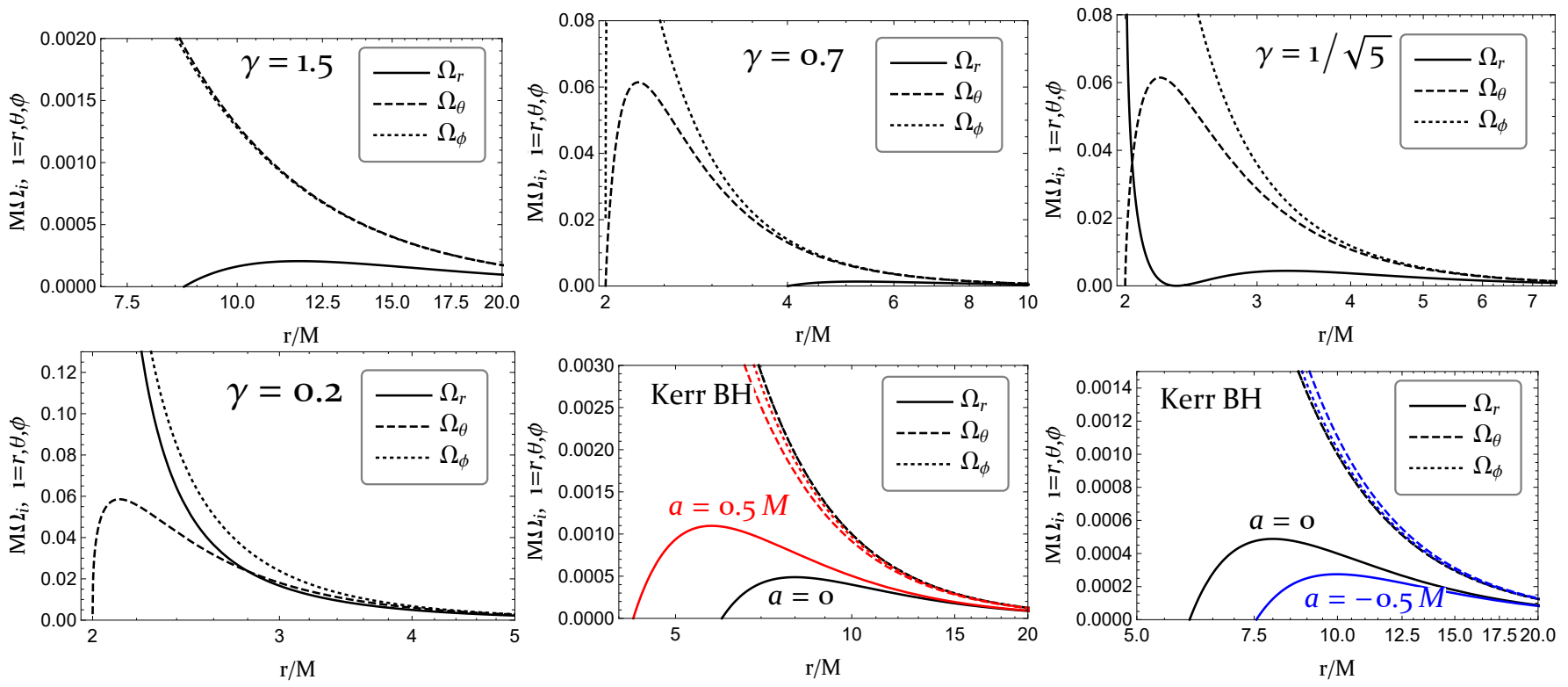

FIG. 3. (color online) Radial profiles of epicyclic frequencies for neutral test particles measured by distant observers in the $\gamma$ space-time for different values of the parameter $\gamma$. The last two figures represent the radial profiles of epicyclic frequencies measured by distant observers for neutral test particles corotating $a / M=0.5$ (red) and counter-rotating $a / M=-0.5$ (blue) around Kerr and Schwarzschild $a=0$ (black) black holes.

$$
\begin{aligned}
& \Omega_{r}^{2}=\Omega_{r, S c h w}^{2}+\frac{M}{r^{4}}\left[4(r-6 M) \log \left(1-\frac{M}{r}\right)+\frac{r^{2}-13 M r+20 M^{2}}{r-2 M}\right] \epsilon+O\left(\epsilon^{2}\right), \\
& \Omega_{\theta}^{2}=\Omega_{\theta, S c h w}^{2}+\frac{M}{r^{3}}\left[4 \log \left(1-\frac{M}{r}\right)+\frac{r-M}{r-2 M}\right] \epsilon+O\left(\epsilon^{2}\right), \\
& \Omega_{\phi}^{2}=\Omega_{\phi, S c h w}^{2}+\frac{M}{r^{3}}\left[2 \log \left(1-\frac{2 M}{r}\right)+\frac{r-M}{r-2 M}\right] \epsilon+O\left(\epsilon^{2}\right),
\end{aligned}
$$

where

$$
\begin{aligned}
& \Omega_{r, S c h w}^{2}=\frac{M(r-6 M)}{r^{4}}, \\
& \Omega_{\theta, S c h w}^{2}=\Omega_{\phi, S c h w}^{2}=\frac{M}{r^{3}},
\end{aligned}
$$

Thus, one can see from Eqs. 48, (49), (50) that oblate (prolate), i.e. values of $\epsilon>0(\epsilon<0)$, deviations from spherical symmetry, i.e. Schwarzschild, increase the values of all components of the epicyclic frequency of test particles.

\section{B. Epicyclic frequencies in Kerr spacetime}

In order to compare epicyclic frequencies of neutral test particles moving along circular orbits in the $\gamma$ space-time with the ones in the Kerr space-time, we briefly review the epicyclic frequencies around a Kerr black hole. In BoyerLindquist coordinates the line element for the Kerr space-time is given by:

$$
\begin{aligned}
d s^{2} & =-\left(1-\frac{2 M r}{\Sigma}\right) d t^{2}+\frac{\Sigma}{\Delta} d r^{2}-2 \frac{2 M r a}{\Sigma} \sin ^{2} \theta d \phi d t \\
& +\Sigma d \theta^{2}+\left(r^{2}+a^{2}+\frac{2 M r a^{2}}{\Sigma} \sin ^{2} \theta\right) d \phi^{2}
\end{aligned}
$$

where

$$
\Sigma=r^{2}+a^{2} \cos ^{2} \theta, \quad \Delta=r^{2}-2 M r+a^{2} .
$$

The comparison between the $\gamma$ and the Kerr space-times is justified by the fact at large distances both metrics tend to become asymptotically flat and so Erez-Rosen as well as BoyerLindquist coordinates tend to become the usual spherical coordinates. Therefore an observer at infinity, measuring some features of the motion of test particles in the accretion disk around a Kerr black hole would be able to compare, at least in principle, the obtained measurements with the expected observations of the corresponding situation in the $\gamma$ space-time. Similarly to the static axially symmetric case, in the stationary case, due to rotation of the source, circular orbits are located on the equatorial plane, thus allowing us to restrict the analysis to the case $\theta_{0}=\pi / 2$. The specific energy, angular momentum and angular velocity of the particle moving on a circular 
orbit around a Kerr black hole are derived from relations $(15)$, (16) and (17), respectively as

$$
\begin{aligned}
& E_{ \pm}=\frac{\sqrt{r}(r-2 M) \pm a \sqrt{M}}{r \sqrt{r \pm 2 a \sqrt{M / r}-3 M}}, \\
& L_{ \pm}= \pm \frac{\sqrt{M}\left(r^{2} \mp 2 a \sqrt{M / r}+a^{2}\right)}{r \sqrt{r \pm 2 a \sqrt{M / r}-3 M}}, \\
& \Omega_{ \pm}= \pm \frac{\sqrt{M}}{a \sqrt{M} \pm r \sqrt{r}} .
\end{aligned}
$$

Where the + and - signs correspond to co-rotating and counter-rotating particles with respect to the direction of rotation of the black hole. Again one can find the radii of circular photon orbits, marginally bound orbits and ISCO from the following relations [29]:

$$
\begin{aligned}
& r_{p s \pm} \pm 2 a \sqrt{M / r_{p s \pm}}-3 M=0 \\
& r_{m b \pm}=2 M \pm a+2 \sqrt{M(M \pm a)} \\
& r_{i s c o \pm}\left(r_{i s c o \pm}-6 M\right) \pm 8 a r_{i s c o \pm} \sqrt{M / r_{i s c o}}-3 a^{2}=0,
\end{aligned}
$$

In Fig. 4 we show the degeneracy between the $\gamma$ metric and the Kerr space-time resulting from measurements of the ISCO or of the photon capture radius. In fact, each given measured value of $r_{i s c o}$ corresponds to one value of $a$ and one value of $\gamma$ showing that the measurement of the ISCO radius alone is not enough to distinguish between the two space-times, unless the value is smaller than $2 M$, as the $\gamma$ space-time cannot have characteristic orbits whose radii are smaller than $2 M$, while rapidly rotating Kerr can. Similar arguments hold for the photon capture radius. However, by observing that the degeneracy in the two cases is different we can conclude that a simultaneous measurement of $r_{i s c o}$ and $r_{p s}$ with enough precision would allow to distinguish between the two geometries around astrophysical compact objects

The epicyclic frequencies, with respect to distant observers, of a test particle moving along circular orbits around a Kerr black hole are given by [20,21]

$$
\begin{aligned}
& \Omega_{r \pm}^{2}=\frac{M\left[r(r-6 M) \pm 8 a r \sqrt{M / r}-3 a^{2}\right]}{r^{2}\left(r^{3} \pm 2 a r^{2} \sqrt{M / r}+a^{2} M\right)}, \\
& \Omega_{\theta \pm}^{2}=\frac{M\left(r^{2} \mp 4 a r \sqrt{M / r}+3 a^{2}\right)}{r^{2}\left(r^{3} \pm 2 a r^{2} \sqrt{M / r}+a^{2} M\right)}, \\
& \Omega_{\phi \pm}^{2}=\frac{M}{r^{3} \pm 2 a r^{2} \sqrt{M / r}+a^{2} M} .
\end{aligned}
$$

In the slow rotation limit, $a \ll M$, the above expressions take the form

$$
\begin{aligned}
& \Omega_{r \pm}^{2}=\Omega_{r, S c h w}^{2} \pm \frac{6(M / r)^{3 / 2}(r+2 M)}{r^{4}} a+O\left(a^{2}\right) \\
& \Omega_{\theta \pm}^{2}=\Omega_{\theta, S c h w}^{2} \mp \frac{6(M / r)^{3 / 2}}{r^{3}} a+O\left(a^{2}\right) \\
& \Omega_{\phi \pm}^{2}=\Omega_{\phi, S c h w}^{2} \mp \frac{2(M / r)^{3 / 2}}{r^{3}} a+O\left(a^{2}\right) .
\end{aligned}
$$

Thus, one can see from (64), (65), (66) that rotation of the spacetime increases (decreases) radial frequency of the harmonic oscillations and decreases (increases) latitudinal and azimuthal frequency of the harmonic oscillations of the corotating (counter-rotating) particle moving along the circular orbit.

This shows that the degeneracy between the Kerr metric and the $\gamma$ metric due to measurements of the value of the ISCO can be broken if one is able to measure simultaneously $\Omega_{r}$ and one between $\Omega_{\theta}$ and $\Omega_{\phi}$.

To this aim, we consider the values of $\gamma$ and $a$ for which the epicyclic frequencies of the neutral test particles have the same value. As we mentioned, if the circular orbits where the particles are moving are located far away from the central object then the two metrics can not be distinguished, since at large distances all frequencies become Newtonian. Therefore, we will focus our attention on relatively short distances. For simplicity we shall also consider slight deviations from the Schwarzschild space-time for both cases, i.e. small deformations $\gamma=1+\epsilon$ with $|\epsilon| \ll 1$, and slow rotation, $|a| \ll M$. Thus, by using the expressions of epicyclic frequencies for the $\gamma$-metric given by Eqs. (48), (49), (50) and for the Kerr black hole, given by Eqs. (64), (65), (66) we find the expressions for the coincidence of all components of the frequencies as

$$
\begin{aligned}
& \Omega_{r, \gamma}^{2}=\Omega_{r, K e r r}^{2} \Rightarrow \frac{a}{\epsilon}=\frac{M\left[4(r-6 M)(r-2 M) \log (1-M / r)+r^{2}-13 M r+20 M^{2}\right]}{\left(r^{2}-4 M^{2}\right)}\left(\frac{r}{M}\right)^{3 / 2} \\
& \Omega_{\theta, \gamma}^{2}=\Omega_{\theta, K e r r}^{2} \Rightarrow \frac{a}{\epsilon}=-\frac{M}{6}\left[4 \log \left(1-\frac{M}{r}\right)+\frac{r-M}{r-2 M}\right]\left(\frac{r}{M}\right)^{3 / 2}, \\
& \Omega_{\phi, \gamma}^{2}=\Omega_{\phi, K e r r}^{2} \Rightarrow \frac{a}{\epsilon}=-\frac{M}{2}\left[2 \log \left(1-\frac{2 M}{r}\right)+\frac{r-M}{r-2 M}\right]\left(\frac{r}{M}\right)^{3 / 2}
\end{aligned}
$$

In Fig. 5 we show the above expressions as functions of the

dimensionless radial coordinate $r / M$. 

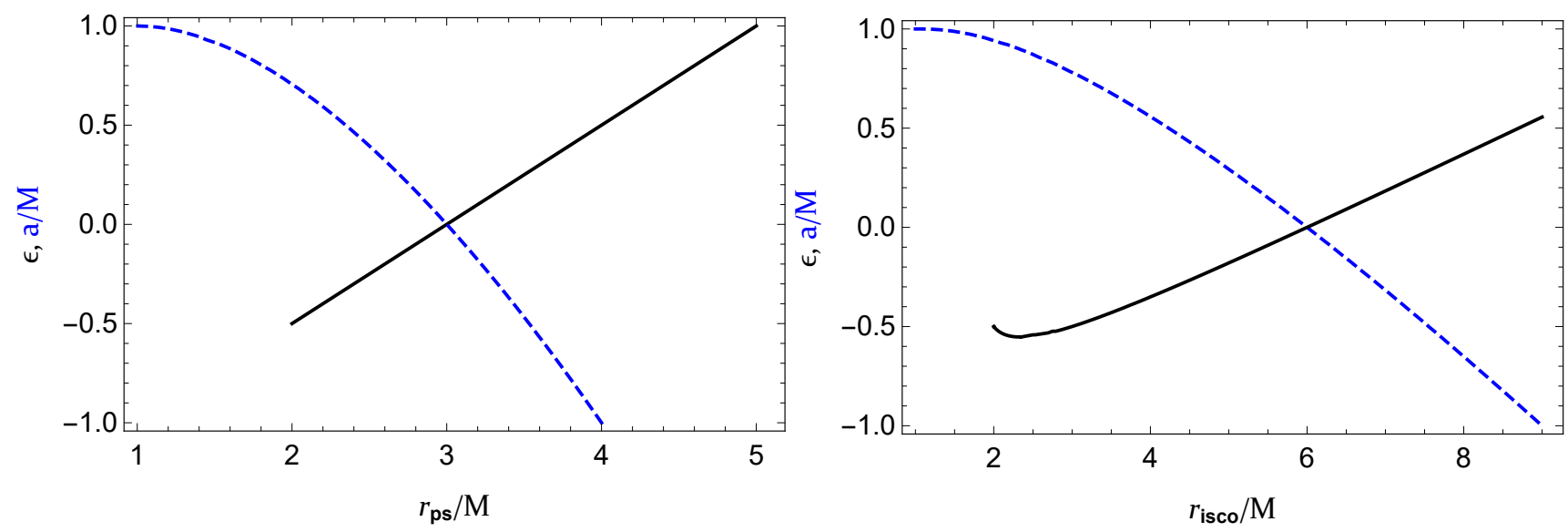

FIG. 4. (Color online) Left panel: Radii of circular photon orbits in $\gamma$ spacetime (black) with $\gamma=1+\epsilon$ and Kerr black hole (blue, dashed). Right panel: ISCO radii of neutral test particles in $\gamma$ spacetime (black) and Kerr black hole (blue, dashed).

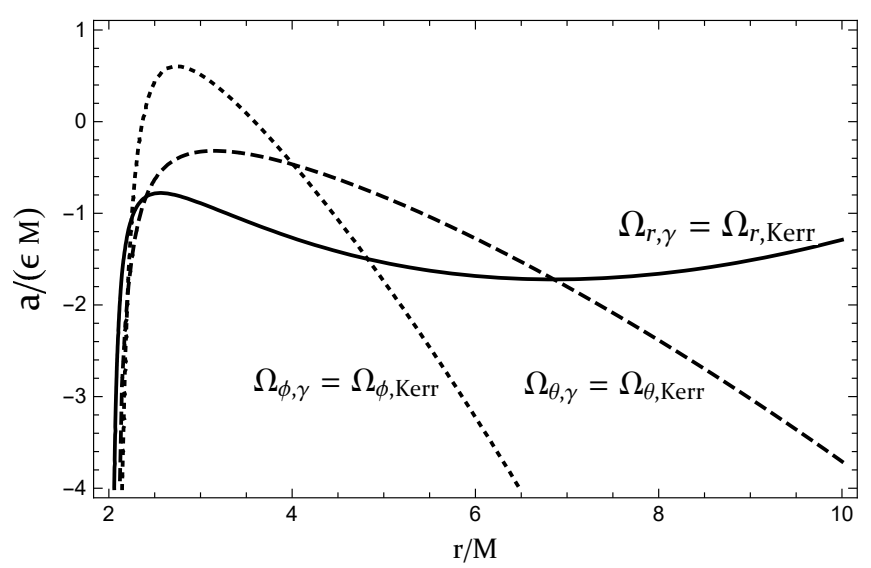

FIG. 5. Radial dependence of ratio $a / \epsilon$ for which the $\gamma$ and Kerr space-times have the same epicyclic frequencies.

Since test particles are oscillating due to slight deviations from stable circular orbits in a space-time which slightly deviates from spherically symmetry, it is useful to focus the attention on the region near $r / M \approx 6$, corresponding to the ISCO for the Schwarzschild metric. One can see from Fig. 5 that the three curves corresponding to the same values for the three frequencies in Kerr and in the $\gamma$ metric intersect in three different points. Therefore, if one measurement was in principle able to measure all three frequencies, the results could easily distinguish the $\gamma$ space-time from Kerr. On the other hand, a measurement of only two frequencies, may not be enough if performed in the vicinity of the point where two curves overlap. For example, the curves obtained from $\Omega_{r}$ and $\Omega_{\theta}$ overlap at $r \approx 6.8 M$ with $a / \epsilon \approx-1.7 M$. Thus, a measurement of these two frequencies in the vicinity of this radius would not be able to distinguish the $\gamma$ space-time from Kerr.

From the above considerations we can derive some conclusion on which kind of objects may or may not be distinguished from a Kerr black hole.

(i) If test particles are co-rotating relative to the Kerr black hole $(a L>0)$ and the source of the $\gamma$ space-time is oblate $(\epsilon>0)$, then the two geometries are distinguishable.

(ii) If test particles are co-rotating relative to the Kerr black hole and the source of the $\gamma$ space-time is prolate $(\epsilon<$ $0)$, then the two geometries may be indistinguishable.

(iii) If test particles are counter-rotating relative to the Kerr black hole $(a L<0)$ and the source of the $\gamma$ space-time is oblate, then the two geometries may be indistinguishable.

(iv) If test particles are counter-rotating relative to the Kerr black hole and the source of the $\gamma$ space-time is prolate, then the two geometries are distinguishable.

Finally, if the latitudinal and radial frequencies of harmonic oscillations of a particle moving along the circular orbit with radius $r=6.8 M$ are detected, one will not be able to distinguish if the central gravitating object is described by the slowly rotating Kerr (and which rotational direction relative to central object is particle moving) or slightly deformed $\gamma$ (and type of deformation) space-times. Since for all three frequencies the ratio $a / \epsilon$ is negative, similar considerations as the ones above apply also to the simultaneous measurement of radial and azimuthal frequencies and to the simultaneous measurement of azimuthal and latitudinal frequencies.

\section{CONCLUSION}

The recent observation of the "black hole shadow" by the Event Horizon Telescope, has opened the door to precise measurements of the geometry in the vicinity of black hole candidates 34. To this day, astrophysical observations of black hole candidates have shown no departure from the relativistic description (see for example the recent measurement of the gravitational redshift of stars orbiting the supermassive black hole candidate at the center of the Milky Way [35]). However such measurements have not been able yet to test 
whether the geometry in the vicinity of black hole candidates is well described by the Kerr metric. For this reason it is useful to study the predictions obtained from different geometries which, while being solutions of Einstein's field equations in vacuum, do not describe a black hole. In this respect the $\gamma$ metric is an ideal candidate due to its simplicity and to its immediate connection to the Schwarzschild space-time.

The $\gamma$ metric describes a deformed Schwarzschild object, it is prolate for $\gamma>1$ and oblate for $\gamma<1$. It turns out that for the former, particle dynamics continues to have the same qualitative features as the Schwarzschild's while for the latter it has much richer structure. In particular, there are two disjoint regions for existence of stable circular orbits for the range $1 / \sqrt{5}<\gamma<1 / 2$ while for $\gamma<1 / \sqrt{5}$, there exist only stable circular orbits. This is exactly like the Newtonian case.

In the present paper we have studied some properties of characteristic circular orbits, i.e. circular null geodesics, marginally bound orbits, stable circular orbits, for massive and massless test particles around the $\gamma$ metric. In particular we focused on the frequencies of harmonic oscillations around stable circular orbits for massive test particles.

We have shown that small deviations from equatorial circular orbits of the particle in the $\gamma$ space-time are described by harmonic oscillations in the radial, vertical, and latitudinal directions. By solving the harmonic oscillator equations we have found the analytical expressions for the frequencies and studied the epicyclic frequencies of particles in the uncoupled orthogonal (radial), vertical (latitudinal) and axial (azimuthal) oscillatory modes relative to comoving and distant observers. The frequencies as measured by distant observers are particularly important since they could, in principle, be measured from the observations of accretion disks around black hole candidates.

We have shown that radial oscillations vanish at the ISCO of the space-time, and below the ISCO the particles fall towards the central object, and no radial oscillations occur. Since at large distances the space-time approaches the Newtonian limit, all epicyclic frequencies tend to the same limit $\Omega_{i}^{2}=M \gamma / r^{3}$ with $i=r, \theta, \phi$. For this reason, we have studied the behaviour of the frequencies in the relativistic regime for slight deviations from spherical symmetry and compared the results with the corresponding frequencies in the slowly rotating Kerr black hole geometry.

We have shown that the two metrics are distinguishable if a simultaneous measurement of all three components of the epicyclic frequencies is available. However, if only two frequencies of oscillations for the test particles are obtained (for example near $r \approx 6.8 M$ for the radial and vertical oscillations) then it may not be possible to distinguish if the geometry around the central object is described by the $\gamma$ or Kerr space-times. Similarly, we have shown that a simultaneous measurement of the radii of the ISCO and the photon capture orbit may be able to distinguish between the two geometries, while the measurement of one radius only is not enough unless such radius is smaller than $2 M$. This is because rapidly rotating Kerr black hole can have orbits below $2 M$ while the $\gamma$ spacetime cannot.

As new an better observations of astrophysical black hole candidates become available, we will soon be able to test the nature of the geometry around such candidates and answer the question whether all such objects must necessarily be described by the Kerr space-time or if nature allows for some other possibility.

\section{ACKNOWLEDGMENTS}

The work was developed under the Nazarbayev University Faculty Development Competitive Research Grant No. 090118FD5348. The authors acknowledge the support of the Ministry of Education of Kazakhstan's target program IRN: BR05236454 and Uzbekistan Ministry for Innovation Development Grants No. VA-FA-F-2-008 and No. YFAFtech-2018-8. N.D. would like to acknowledge Nazarbayev University, Astana, Kazakhstan for the hospitality and Albert Einstein Institute, Golm, Germany for a visit that partially facilitated this work. D.M. and N.D. wish to express their gratitude to Bobomurat Ahmedov for useful discussion on the properties of the gamma metric and acknowledge the Ulugh Beg Astronomical Institute, Tashkent, Uzbekistan for the hospitality through Abdus Salam International Centre for Theoretical Physics through Grant No. OEA-NT-01.
[1] D. M. Zipoy, J. Math. Phys. 7, 1137 (1966).

[2] B. H. Voorhees, Phys. Rev. D 2, 2119 (1970),

[3] K. S. Virbhadra, arXiv e-prints , gr-qc/9606004 (1996), arXiv:gr-qc/9606004 [astro-ph].

[4] D. Papadopoulos, B. Stewart, and L. Witten, Phys. Rev. D 24, 320 (1981).

[5] L. Herrera, F. M. Paiva, and N. O. Santos, J. Math. Phys. 40, 4064 (1999), gr-qc/9810079.

[6] L. Herrera and J. L. H. Pastora, I. Math. Phys. 41, 7544 (2000) gr-qc/0010003.

[7] J. L. Hernández-Pastora and J. Martín, Gen. Rel. Grav. 26, 877 (1994).

[8] W. B. Bonnor, General Relativity and Gravitation 24, 551 (1992).
[9] W. C. Hernandez, Phys. Rev. 153, 1359 (1967)

[10] B. W. Stewart. D. Papadopoulos, L. Witten, R. Berezdivin, and L. Herrera, Gen. Rel. Grav. 14, 97 (1982)

[11] L. Herrera, G. Magli, and D. Malafarina, Gen. Rel. Grav. 37, 1371 (2005), gr-qc/0407037.

[12] A. N. Chowdhury, M. Patil, D. Malafarina, and P. S. Joshi, Phys. Rev. D 85, 104031 (2012), arXiv:1112.2522 [gr-qc].

[13] K. Boshkayev, E. Gasperín, A. C. Gutiérrez-Piñeres, H. Quevedo, and S. Toktarbay, Phys. Rev. D 93, 024024 (2016), arXiv: 1509.03827 [gr-qc]

[14] C. A. Benavides-Gallego, A. Abdujabbarov, D. Malafarina, B. Ahmedov, and C. Bambi, Phys. Rev. D 99, 044012 (2019), arXiv: 1812.04846 [gr-qc] 
[15] A. B. Abdikamalov, A. A. Abdujabbarov, D. Ayzenberg, D. Malafarina, C. Bambi, and B. Ahmedov, arXiv e-prints, arXiv:1904.06207 (2019), arXiv:1904.06207 [gr-qc].

[16] M. A. Abramowicz and W. Kluźniak, Astrophysics and Space Science 300, 127 (2005), arXiv:astro-ph/0411709 [astro-ph].

[17] M. Kološ, Z. Stuchlík, and A. Tursunov, Classical and Quantum Gravity 32, 165009 (2015), arXiv:1506.06799 [gr-qc]

[18] M. Kološ, A. Tursunov, and Z. Stuchlík, Eur. Phys. J. C 77, 860 (2017), arXiv: 1707.02224 [astro-ph.HE].

[19] A. Tursunov, Z. Stuchlík, and M. Kološ, Phys. Rev. D 93, 084012 (2016), arXiv:1603.07264 [gr-qc].

[20] G. Török and Z. Stuchlík, Astronomy and Astrophysics 437, 775 (2005), arXiv:astro-ph/0502127 [astro-ph].

[21] G. Török, M. A. Abramowicz, W. Kluźniak, and Z. Stuchlík, Astronomy and Astrophysics 436, 1 (2005).

[22] C. Bambi. I. Cosmol. Astropart. Phys. 9, 014 (2012) arXiv: 1205.6348 [gr-qc]

[23] C. Bambi and S. Nampalliwar, Europhys. Lett. 116, 30006 (2016), arXiv:1604.02643 [gr-qc]

[24] Z. Stuchlík and M. Kološ, MNRAS 451, 2575 (2015) arXiv:1603.07339 [astro-ph.HE].
[25] Z. Stuchlík and A. Kotrlová, Gen. Relativ. Gravitation 41, 1305 (2009), arXiv:0812.5066 [astro-ph].

[26] Z. Stuchlík and M. Kološ. Astrophys. J. 825, 13 (2016), arXiv:1608.01659 [astro-ph.HE].

[27] Z. Stuchlík and M. Kološ. Astronomy and Astrophysics 586, A130 (2016), arXiv:1603.07366 [astro-ph.HE]

[28] G. Erez and N. Rosen, Bull. Res. Council Israel 8F, 47 (1959).

[29] J. M. Bardeen, W. H. Press, and S. A. Teukolsky, Astrophysical Journal 178, 347 (1972).

[30] S. Hod, Phys. Rev. D 88, 087502 (2013).

[31] L. Herrera, Found. Phys. Lett. 18, 21 (2005), gr-qc/0402052.

[32] B. Toshmatov, Z. Stuchlík, and B. Ahmedov, Phys. Rev. D 95, 084037 (2017), arXiv:1704.07300 [gr-qc].

[33] K. A. Boshkayev, M. Muccino, J. A. Rueda, and G. D. Zhumakhanova, arXiv e-prints , arXiv:1802.06773 (2018), arXiv: 1802.06773 [astro-ph.HE].

[34] Event Horizon Telescope Collaboration, K. Akiyama, and et al., Astrophys. J. Lett. 875, L1 (2019).

[35] Gravity Collaboration, R. Abuter, and et al., Astronomy \& Astrophysics 615, L15 (2018), arXiv:1807.09409 [astro-ph.GA]. 